\title{
Injection scheme with deflecting cavity for a fourth-generation storage ring
}

\author{
J. Kim, ${ }^{1}$ G. Jang, ${ }^{1}$ M. Yoon, ${ }^{1}$ B-H. Oh,${ }^{2}$ J. Lee, ${ }^{2}$ J. Ko, ${ }^{2}$ Y. Parc, ${ }^{2}$ \\ T. Ha, ${ }^{2}$ D. Kim, ${ }^{2}$ S. Kim, ${ }^{3, *}$ and S. Shin ${ }^{2, \dagger}$ \\ ${ }^{1}$ Department of Physics, POSTECH, Pohang, Gyungbuk 37673, Korea \\ ${ }^{2}$ Pohang Accelerator Laboratory, POSTECH, Pohang, Gyungbuk 37673, Korea \\ ${ }^{3}$ FRIB, MSU, East Lansing, Michigan 48824, USA
}

(Received 7 August 2018; published 14 January 2019)

\begin{abstract}
We suggest a new on-axis injection scheme that uses a transverse deflecting rf cavity to kick the incoming beam into an already populated bucket but with a timing offset from the synchronous phase. In a new on-axis injection scheme, two deflecting rf cavities are required: one upstream of the injection point that crabs the stored beam and the other downstream of the injection point that both uncrabs the stored beam and kicks the incoming beam onto the axis of the orbital plane. We present a theoretical analysis and numerical simulations of the stored beam and injected beam with the new injection scheme.
\end{abstract}

DOI: 10.1103/PhysRevAccelBeams.22.011601

\section{INTRODUCTION}

The fourth-generation storage ring (4GSR) based on the multibend achromat (MBA) lattice concept may be able to surpass the brightness and coherence that are attained using present third-generation storage rings (3GSRs). MAX-IV [1] was the first MBA machine; its successor, Sirius [2], is currently under construction. Other projects are being conducted to convert existing $3 \mathrm{G}$ machines such as ESRF, APS, SPring-8, and ALS [3-6] to 4GSRs. In these facilities, beam emittance decreases to a few hundred picometers or even to $\leq 100 \mathrm{pm}$.

To suppress the dispersions caused by the bending magnets, the 4GSR requires stronger quadrupoles than the 3GSR does, so large negative natural chromaticities occur in both transverse planes. To correct them, strong chromatic sextupoles are needed. These nonlinear elements will significantly reduce the dynamic apertures, and this is a great challenge for 4GSR lattice design. These nonlinear elements will significantly reduce the dynamic apertures down to $\sim 2 \mathrm{~mm}$ for many 4GSR lattice designs [4-6], leading to severe difficulties for injection $[7,8]$. Therefore, when the emittance of a storage ring is pushed to an extremely small value (i.e., $\leq \sim 100 \mathrm{pm}$ ), the dynamic aperture becomes insufficient to enable accumulationbased injection, so only on-axis injections are workable for such a very small dynamic aperture.

\footnotetext{
*kims@frib.msu.edu

†tlssh@postech.ac.kr
}

Published by the American Physical Society under the terms of the Creative Commons Attribution 4.0 International license. Further distribution of this work must maintain attribution to the author(s) and the published article's title, journal citation, and DOI.
So far, three on-axis injection schemes have been proposed for 4GSR. (i) "Swap-out" injection [9] uses a fast dipole kicker to inject a fresh high-charge beam onto the closed orbit while the stored beam is extracted. (ii) Longitudinal injection with a low-frequency rf system injects a bunch on axis at an $\mathrm{rf}$ phase that is far away from the synchrotron phase and with an energy slightly higher than the stored beam; the synchrotron oscillation of the injected beam is damped to the synchrotron phase. (iii) Longitudinal injection with a double-frequency rf system alters two rf voltages to create empty rf buckets that can be taken for onaxis injection; after bunches are injected, the voltage-altering process is reversed, and the injected bunches can be transferred longitudinally to the main rf buckets. These new injection schemes suggest solutions for beam injection into a small dynamics aperture, but unfortunately the schemes require a tight condition on the injected beam and a kicker that has a very short pulse to avoid disturbing the stored beam.

This paper presents a new on-axis injection scheme for the storage ring with a small dynamic aperture. The scheme uses two transverse deflecting cavities (TDCs) and thereby eliminates the need to swap out stored beams and implement a short pulse (a few nanoseconds) kicker. This scheme has the additional benefit of generating a short $\mathrm{x}$-ray pulse between the deflecting cavities. Section II introduces the scheme for on-axis injection with two deflecting cavities. Section III shows simulation results for the stored beam and injected beam in the lattice of the PAL-4GSR. Section IV analyzes the result, and Sec. V presents conclusions.

\section{ON-AXIS INJECTION WITH TRANSVERSE DEFLECTING CAVITY}

The longitudinal motion can be described using the longitudinal equation of motion for a relativistic particle 


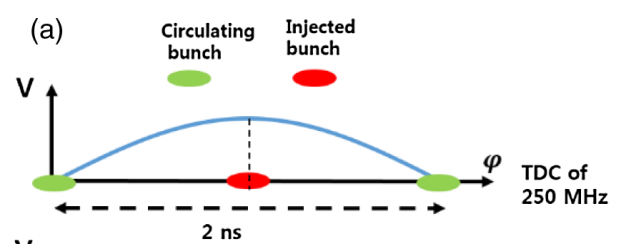

(b)
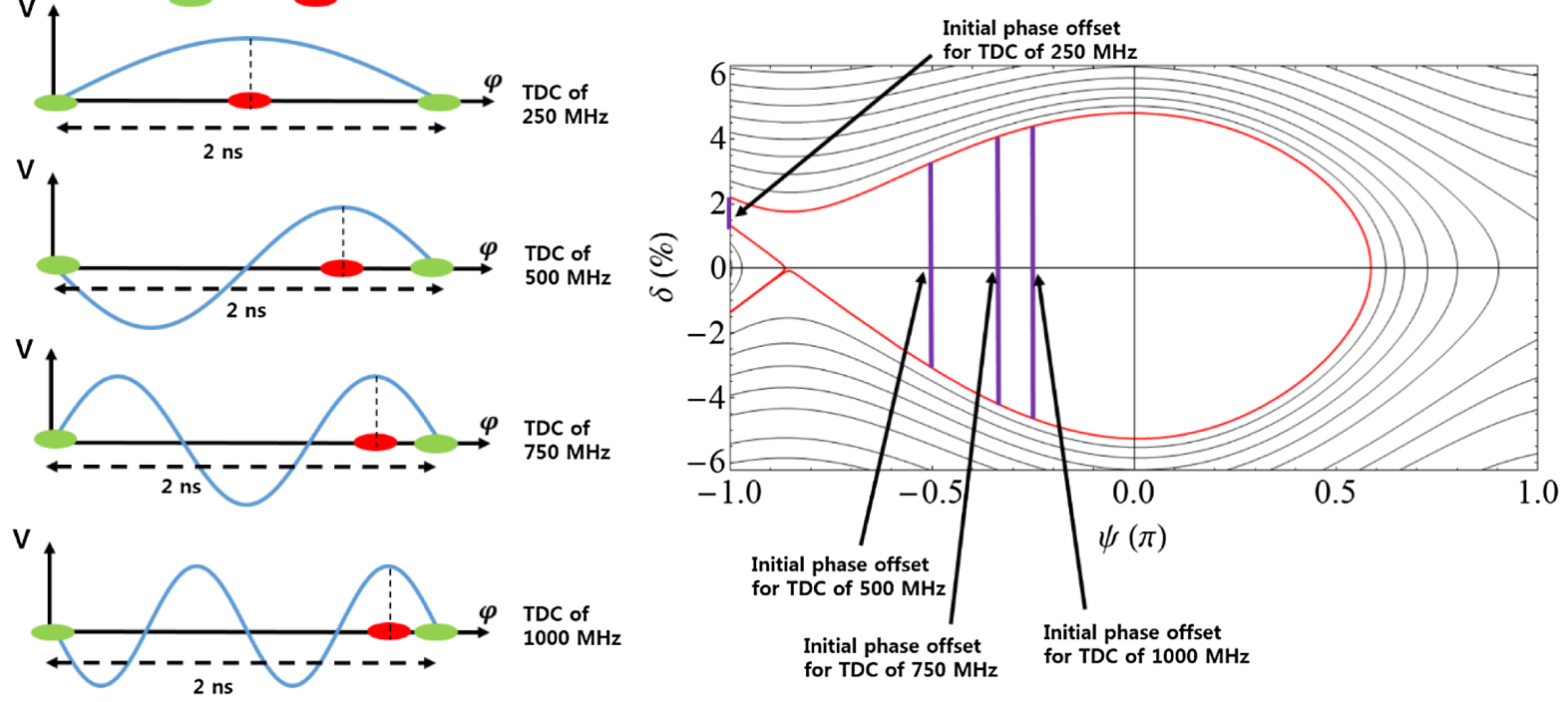

FIG. 1. (a) Working conditions along the rf frequency of the deflecting cavity. (b) Timing offset (phase offset) and energy acceptance of the injected beam along the rf frequency of the deflecting cavity. The energy acceptance at $750 \mathrm{MHz}$ frequency is already close to the energy acceptance at synchrotron phase for an injected beam.

$$
\begin{gathered}
\frac{\partial \varphi}{\partial t}=\omega_{r f} \alpha_{c} \delta, \\
\frac{\partial \delta}{\partial t}=\frac{1}{E_{0} T_{0}}\left[e V_{r f}-U(\delta)\right],
\end{gathered}
$$

where $\varphi$ is the phase with the given $\mathrm{rf}$ frequency, $\delta$ is the relative energy deviation, $E_{0}$ is the nominal energy, $T_{0}$ is the revolution period, $V_{r f}$ is the rf voltage, and $\omega_{r f}$ is the angular frequency of the ring. $\alpha_{c}$ is the first-order momentum compaction factor; $U(\delta)$ is the synchrotron radiation energy loss per turn to first order in $\delta$, and its derivation can be found in [10]

$$
U(\delta)=U_{0}\left(1+J_{\delta} \delta\right)
$$

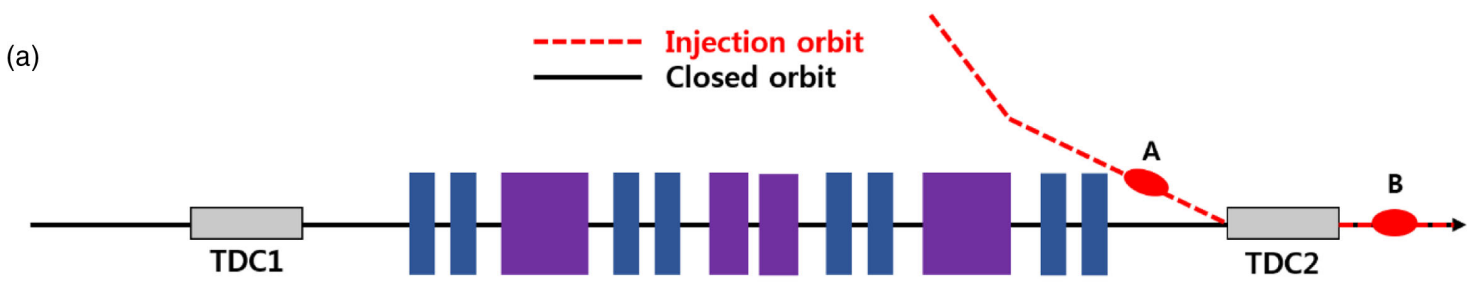

(b)
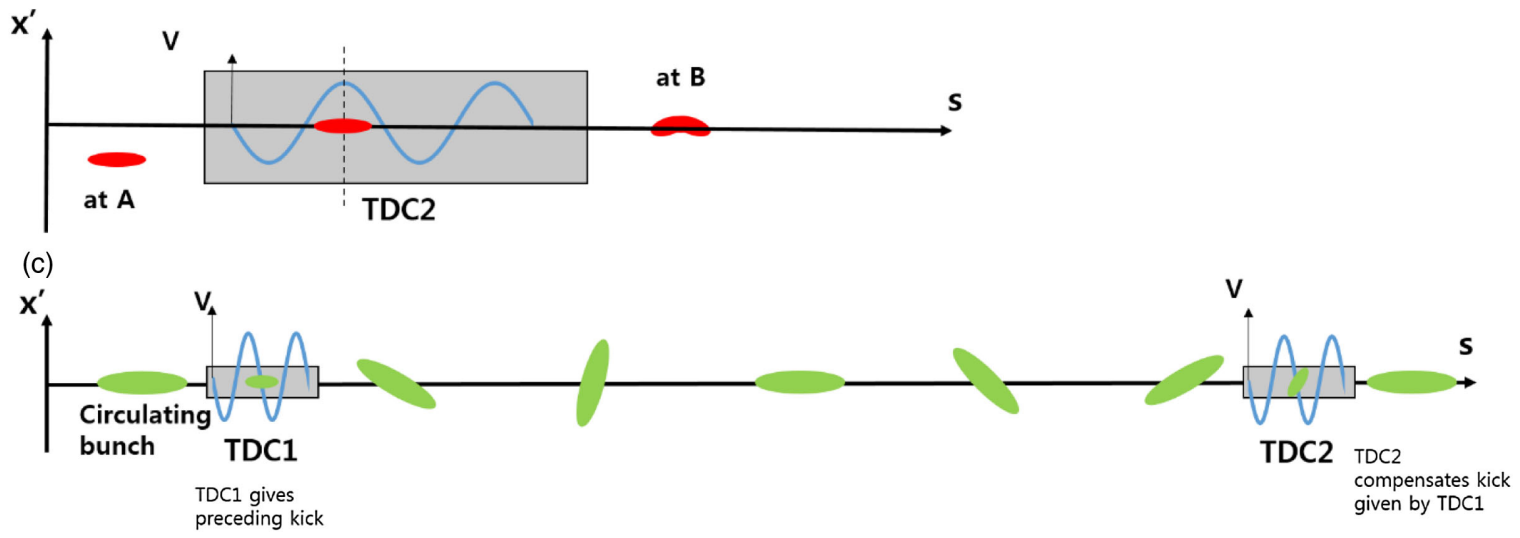

FIG. 2. Schematics for the working principle of on-axis injection with two deflecting cavities. (a)-(b) The second TDC kicks the injected beam on-axis. (c) The two TDCs crab and uncrab the stored beam (circulating bunch). 
TABLE I. Deflecting cavity parameters.

\begin{tabular}{lcc}
\hline \hline Parameter & Value & Unit \\
\hline Frequency & 750 & $\mathrm{MHz}$ \\
Deflecting voltage & 4.5 (total) & $\mathrm{MV}$ \\
Cavity type & Normal conducting & $\ldots$ \\
Loaded Q & 3000 & $\ldots$ \\
Pulse duration & 2 & $\mu \mathrm{s}$ \\
Repetition rate & 50 & $\mathrm{~Hz}$ \\
\hline \hline
\end{tabular}

where

$$
J_{\delta}=2+\frac{\oint\left(\frac{\eta_{x}}{R^{3}}+2 \eta_{x} \frac{K_{1}}{R}\right) d s}{\oint \frac{1}{R^{2}} d s}
$$

and the fractional part in Eq. (4) is calculated from the bending magnet, where $R$ is the bending radius, $\eta_{x}$ is the horizontal dispersion function, and $K_{1}$ is the usual focusing parameter [10]. Equation (1) can be used to calculate the acceptance of longitudinal phase space [Fig. 1(b), red line].

The previous longitudinal injection scheme [11] needs (i) a kicker that injects a short pulse (a few nanoseconds) so that the stored beam is not disturbed and (ii) tight energy jitter and a small energy spread of the injected beam so that the injected beam is put within the acceptance of the longitudinal phase space at the phase $-\pi$. To overcome these stringent requirements, a new on-axis injection scheme with two transverse deflecting cavities is suggested (Fig. 2). An injected beam with an angle is kicked by the second TDC to be put in the same position as the stored beam in transverse phase space. The phase of the TDC is on crest for the injected beam and zero crossing for the stored beam. The two TDCs work for the stored beam as a system to generate short x-ray pulses [12-15] that are used to tilt the stored beam by the first TDC and to remove the tilt of the stored beam by the second TDC. This scheme does not use a dipole kicker with a shorter pulse than the bunch spacing.

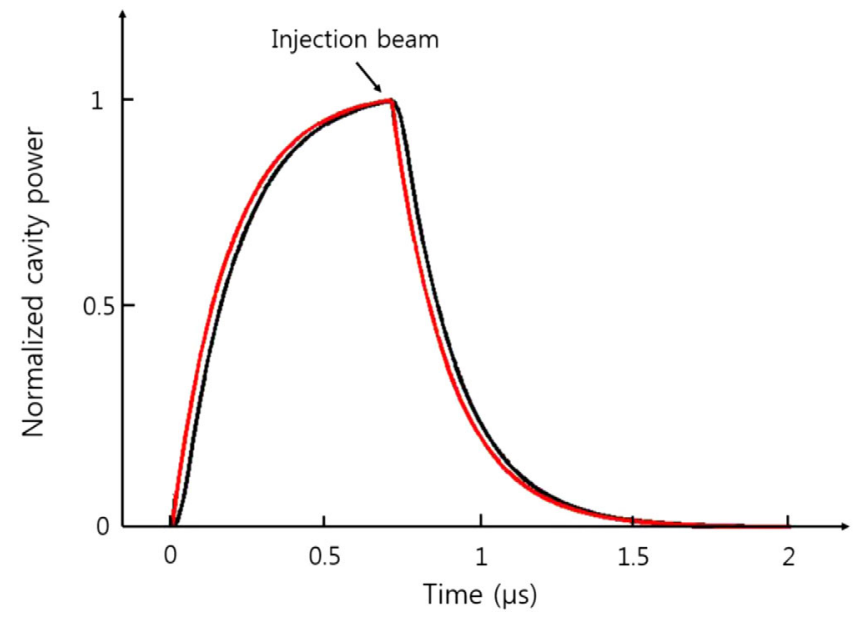

FIG. 3. Pulse shape of normalized cavity power. Red, ideal square input. Black, 100 ns ramp.
TABLE II. Parameters relevant to the PAL-4GSR lattice.

\begin{tabular}{lcc}
\hline \hline Parameter & Value & Unit \\
\hline Energy & 3 & $\mathrm{GeV}$ \\
Emittance (flat/round) & $90 / 58$ & $\mathrm{pm}$ \\
Circumference & 569.96 & $\mathrm{~m}$ \\
Tune (x/y) & $47.545 / 18.203$ & $\ldots$ \\
Natural chromaticity (x/y) & $-96.0 /-57.9$ & $\ldots$ \\
Radiation loss per turn & 0.468 (without ID) & $\mathrm{MeV}$ \\
Momentum compaction & $1.45 \times 10^{-4}$ & \\
Damping partition(H/V/L) & $1.82 / 1.00 / 1.18$ & \\
Damping time (H/V/L) & $13.37 / 24.35 / 20.66$ & $\mathrm{~ms}$ \\
\hline \hline
\end{tabular}

The rf frequency of the TDC is related to the timing offset of the injected beam from the synchrotron phase (Fig. 1). This new on-axis injection scheme must work at $\omega_{\mathrm{TDC}}=n \times\left(\omega_{M} / 2\right)$, where $\omega_{\mathrm{TDC}}$ is the rf frequency of the TDC and $\omega_{M}$ is the rf frequency of the main cavity. All rf frequencies larger than $250 \mathrm{MHz}$ for the TDC provide broader energy and timing acceptances for the injected beam compared to the previous longitudinal injection scheme [11] (Fig. 1). An increase in rf frequency reduces rf input power but increases a nonlinear effect that causes leakage on compensation for the stored beam. This relationship will be discussed later. Considering the available rf power and acceptable leakage on compensation for the stored beam, $750 \mathrm{MHz}$ was selected as the rf frequency for the TDC.

The rf parameters of the deflecting cavity (Table I) were chosen based on the result of a model of a three-cell deflecting cavity with heavy wakefield damping and internal loads [16]. To give a 1.5-mrad transverse kick to an injected beam with a $2-\mu$ s pulse (Fig. 3), a normal conducting rf cavity with loaded $Q \sim 3000$ was chosen. The deflecting voltage per cavity is $2.25 \mathrm{MV}$, and two cavities as the second TDC are used to kick the injected beam and to compensate for the tilt on the stored beam. The turn spacing of an injected beam in the PAL-4GSR is

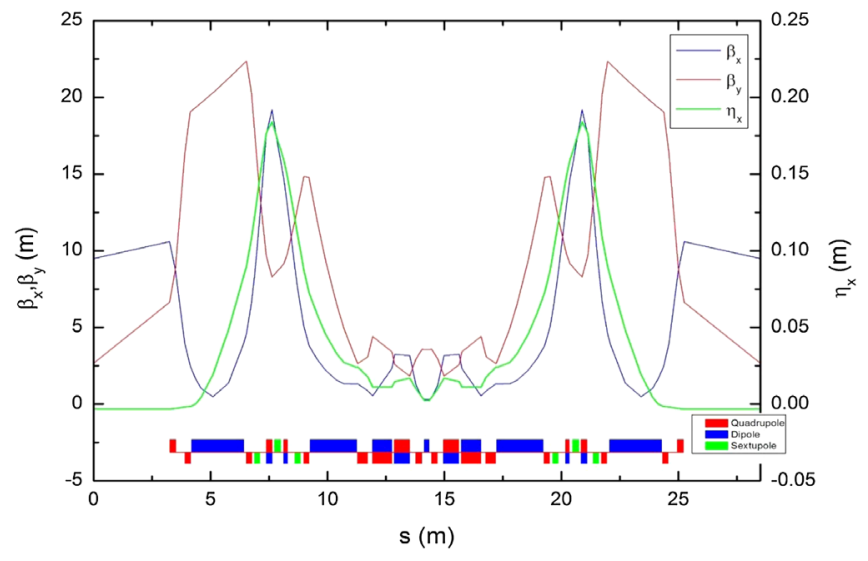

FIG. 4. Lattice functions for PAL-4GSR. 


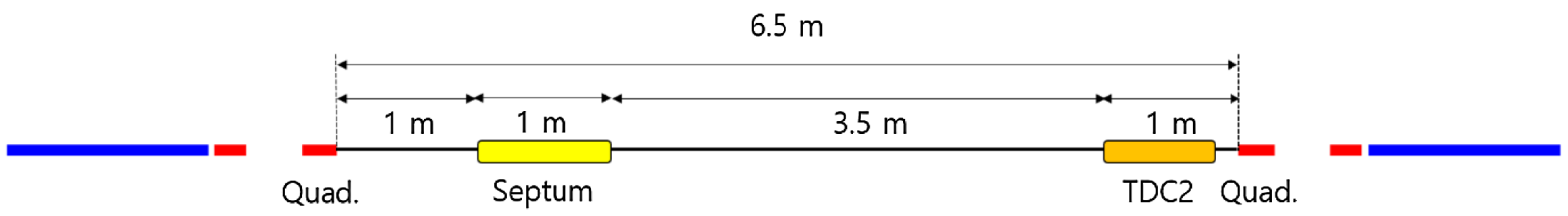

FIG. 5. Layout of the injection element. TDC1 is located in the straight section upstream of the injection region. Blue and red indicate the bending magnet and the quadrupole magnet, respectively.

$\sim 2 \mu \mathrm{s}$. The rf should be a pulsed rf with the repetition rate of $50 \mathrm{~Hz}$. Here the reason to choose the high repetition rate of $50 \mathrm{~Hz}$ rather than a general injection repetition rate of a few hertz is for using a short pulse $\mathrm{x}$ ray from the tilted stored beam at a bending beam line. Another two cavities as the first TDC are used to tilt the stored beam in the upstream section. A feasible option that complies with these specifications is the normal conducting deflecting cavity studied for short pulse X-ray generation at Argonne National Laboratory in collaboration with SLAC National Accelerator Laboratory, the frequency of which is scaled from 2856 to $750 \mathrm{MHz}$ [16]. In this case, the peak rf power required for $4.5 \mathrm{MV}$ deflecting voltage will be approximately $55 \mathrm{MW}$. (This is the case of one rf cavity for TDC1 or TDC2. In the case of two rf cavities for TDC1 or TDC2, $14 \mathrm{MW}$ is required for $4.5 \mathrm{MV}$ deflecting voltage.) This will be operated with $\sim 1 \mu \mathrm{s}(\mathrm{FWHM}) / 50 \mathrm{~Hz}$ pulses.

\section{NUMERICAL SIMULATION WITH PAL-4GSR LATTICE}

A numerical simulation of the new injection scheme with a deflecting cavity was conducted. In the simulation, the scheme was applied to the lattice of the PAL-4GSR $3 \mathrm{GeV}$ storage ring [17]. The PAL-4GSR storage ring is a hybrid seven-bend achromat (H7BA) lattice with a horizontal emittance of $90 \mathrm{pm}$. The ring with $570 \mathrm{~m}$ circumference is composed of 20 symmetrical cells. The length of the straight section is considered as $6.5 \mathrm{~m}$ to accommodate two SCRF modules from the experience on PLS-II [18]. The PAL-4GSR lattice contains a $2 \mathrm{~T}$ superbend in the central dipole to produce radiation with a critical energy of $12 \mathrm{keV}$. The relevant parameters are listed in Table II, and the lattice functions are shown in Fig. 4.

The concept of ESRF-II and APSU lattices was adopted in the PAL-4GSR lattice. The dispersion was deliberately enlarged between the first and second dipoles and between the sixth and seventh dipoles. And all chromatic sextupoles were located in this dispersion bump region to reduce the strength required to control the chromaticity. The betatron phase advances between the two dispersion bumps were set to $\Delta \varphi_{x} \sim 3 \pi$ in the horizontal plane and $\Delta \varphi_{y} \sim \pi$ in the vertical plane, respectively. As a result, nonchromatic effects of the sextupoles are canceled out naturally. To minimize natural emittance, five-step longitudinal gradient dipoles and reverse bending magnets were considered.
The storage ring rf system includes two SC cavities, each one driven by a klystron delivering up to $240 \mathrm{~kW} \mathrm{CW}$ at $500 \mathrm{MHz}$. The accelerating voltage in the main rf cavity is 2.15 MV to realize a bucket height of $\sim 5 \%$. The stored beam has a rms bunch length $=8 \mathrm{~mm}$ with the assumption of a third harmonic cavity for bunch prolongation and natural energy spread $=0.1 \%$. When the beam current is $400 \mathrm{~mA}$ in the multibunch operation mode, the emittance increase due to intrabeam scattering is $10 \%$ in the roundbeam mode.

The injection layout is shown in Fig. 5. The in-vacuum septum magnet [19] deflects the injected beam by $150 \mathrm{mrad}$ from the beam transport line, and after the septum magnet, the injected beam has $6 \mathrm{~mm}$ offset from the stored beam and $1.5 \mathrm{mrad}$ angle. Then the injected beam is assumed to have zero offset and zero angle after the second deflecting cavity, and, finally, transverse on-axis injection is realized.

To explore the transverse injection dynamics in detail, we used the particle-tracking code ELEGANT [20]. In the tracking, we launched 2000 particles with the beam distribution from the existing PLS-II linear accelerator (Table III). Injected bunches with the timing offset were successfully damped to the synchronous phase in the $\mathrm{rf}$ bucket (Fig. 6). The large transverse emittance $(58 \mathrm{~nm})$ of the injected beam was damped toward natural emittance (90 pm) after 40000 turns.

Emittance degradation of the stored beam was also examined. First of all, a perfect machine was assumed to investigate those effects. For the simulation, we use the extreme case of highest voltage 4.5 MV for the deflecting cavity (Fig. 3). Of course, the effects diminished as the voltage is reduced. Particle distributions in horizontal phase space and horizontal momentum-to-time space (Fig. 7) were simulated with the deflecting cavity on and the deflecting cavity off. In the case of a perfect machine, $30 \%$ emittance degradation occurred.

TABLE III. Initial particle distribution.

\begin{tabular}{lcc}
\hline \hline Parameter & Value & Unit \\
\hline Horizontal emittance & 58 & $\mathrm{~nm}$ \\
Vertical emittance & 58 & $\mathrm{~nm}$ \\
Energy spread (rms) & 0.2 & $\%$ \\
Bunch length (rms) & 20 & $\mathrm{ps}$ \\
Beta function at IP (x/y) & $6.46 / 4.32$ & $\mathrm{~m}$ \\
\hline \hline
\end{tabular}



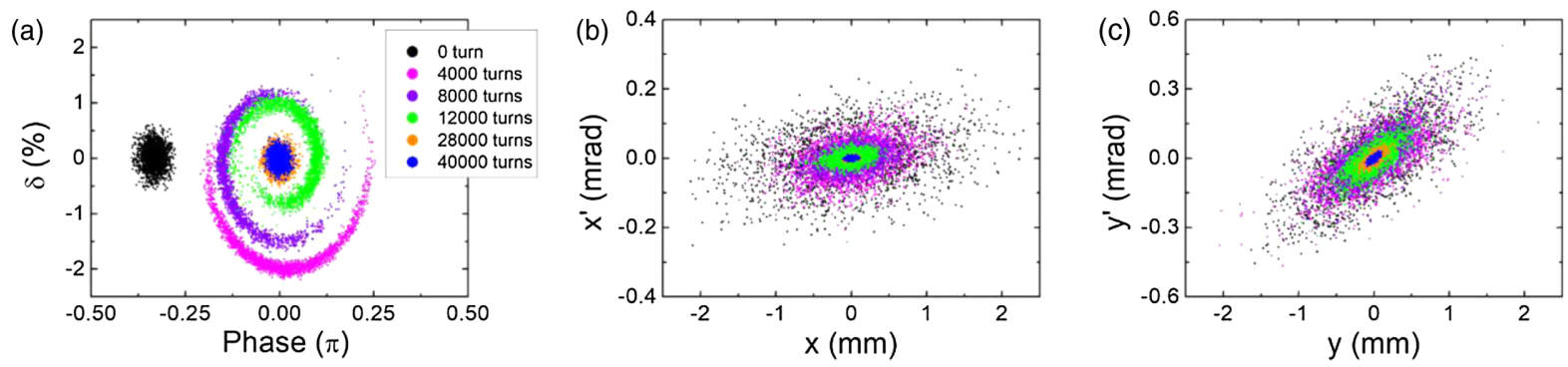

FIG. 6. Particle distributions in the longitudinal (a), horizontal (b), and vertical (c) planes with up to 40000 turns following the injection.

\section{ANALYSIS ON EMITTANCE DEGRADATION}

This section presents an analysis of emittance degradation in the perfect machine and introduces the effects that arise in a real machine with errors. The location of TDC2 was fixed in consideration of the injection septum and injected orbit, and then the position of TDC1 was located to have $n \pi$ phase advance between TDC1 and TDC 2 so that I or -I transformation is satisfied. In the PAL-4GSR lattice, a phase advance of $5 \pi$, which corresponds to $-\mathrm{I}$ transformation, is set between the two TDCs. However, cancellation was not perfect in either the position coordinate (x) or the momentum coordinate (x') (Fig. 7).
An analysis of the transfer map between TDC1 and TDC2 can explain the imperfect cancellation. With a thirdorder transfer map, the beam coordinates at TDC 2 can be written as

$x_{i}=\sum R_{i j} x_{j 0}+\sum T_{i j k} x_{j 0} x_{k 0}+\sum U_{i j k l} x_{j 0} x_{k 0} x_{l 0}$,

where coordinates with subscript 0 are values at TDC1. First, the linear terms were checked. The value of $R_{22}$ was $-0.9883 \mathrm{rad} / \mathrm{rad}$, whereas perfect cancellation requires $R_{22}=-1$. This deviation of $R_{22}$ from -1 is the source of linearly correlated residual motion in horizontal phase
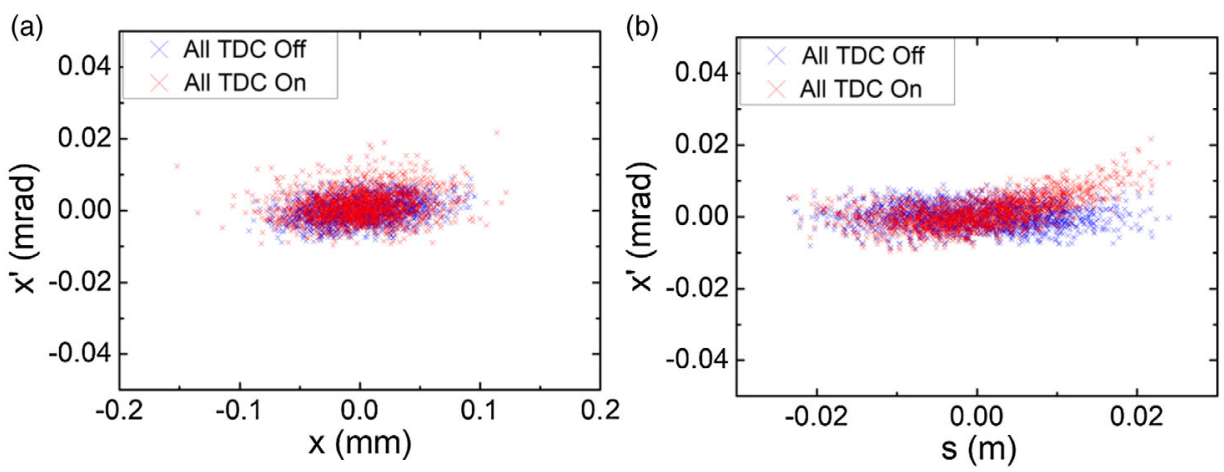

FIG. 7. Particle distributions in the horizontal phase space (a) and horizontal momentum via phase (b) after the second TDC.
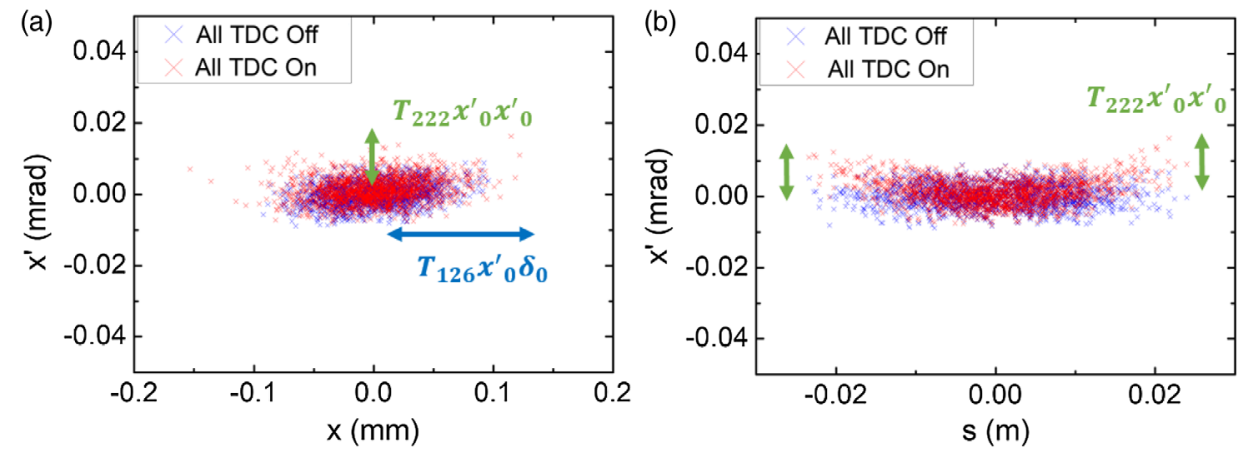

FIG. 8. Particle distributions in the horizontal phase space (a) and horizontal momentum via phase (b) after the second TDC. In this case, the TDC1 voltage is adjusted to get the minimum emittance degrade. 


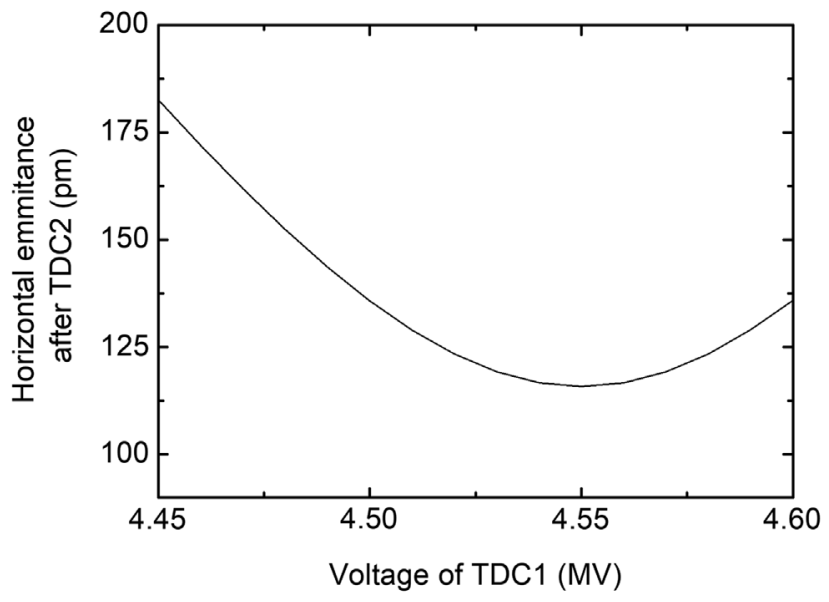

FIG. 9. Horizontal emittance after TDC2 along the voltage of TDC1. Here the TDC2 voltage is fixed with $4.5 \mathrm{MV}$.

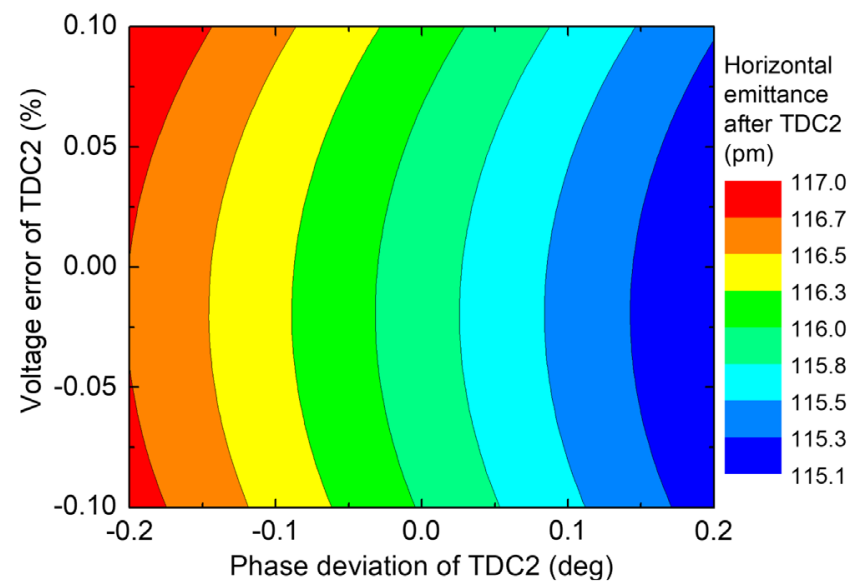

FIG. 10. Horizontal emittance after TDC2 along the relative phase error and voltage error between TDC1 and TDC2.

space; to suppress this motion, the voltage of TDC1 is adjusted to manipulate the momentum coordinate in the initial horizontal phase space. This change improved the cancellation of linearly correlated residual motion (Fig. 8). At a TDC1 voltage of $4.55 \mathrm{MV}$, horizontal emittance after

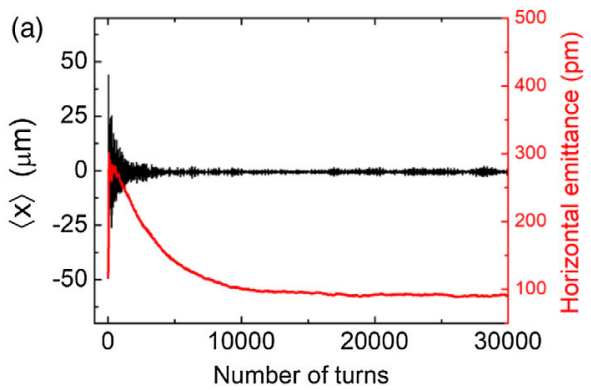

TDC2 had the minimal value 117 pm (Fig. 9). The TDC voltage ratio $4.50 / 4.55(\mathrm{TDC} 2 / \mathrm{TDC} 1)=0.9890$ is close to the $R_{22}$ ratio $0.9883 / 1$.

Among the nonlinear terms of the transfer map, coefficients $T_{126}$ and $T_{222}$ are dominant. Their values are $78.53 \mathrm{~m} / \mathrm{rad}$ and $31.27 \mathrm{rad} / \mathrm{rad}^{2}$, respectively. Their impacts on the stored beam after TDC2, which can be calculated by $T_{126} x_{0}^{\prime} \delta_{0}$ in the position coordinate and by $T_{222} x_{0}^{\prime} x_{0}^{\prime}$ in the momentum coordinate, explains the residual diffusion after TDC2 (Fig. 8). Specifically, a particle having $\delta_{0}=0.003$ (at the edge of a 3- $\sigma$ cut under $0.1 \%$ momentum spread) and $x_{0}^{\prime}=0.6 \mathrm{mrad}$ (maximum kick received by TDC1, at tails of the stored beam) diffuses in the position coordinate as much as $0.141 \mathrm{~mm}$ after TDC2. Also, a particle having $x_{0}^{\prime}=0.6 \mathrm{mrad}$ diffuses in the momentum coordinate as much as $0.011 \mathrm{mrad}$ after TDC2. These analyses indicate that the cancellation after TDC2 is also dependent on the bunch length, voltage of the TDC, and frequency of the TDC.

The relationship between rf errors and emittance growth, which specifies rf tolerances, is investigated. Errors of voltage and phase between TDCs are straightforwardly swept as the source of $\mathrm{rf}$ errors. The result shows that emittance growth just after TDC2 is negligible within boundaries of $\pm 0.2^{\circ}$ phase errors and $\pm 0.1 \%$ voltage errors (Fig. 10). In addition, centroid motion and emittance growth along turn by turn is numerically calculated for two error sets [(a) rf error with $0.2^{\circ}$ phase deviation and $0.1 \%$ voltage error and (b) rf error with $-0.2^{\circ}$ phase deviation and $-0.1 \%$ voltage error], lying on the error boundaries (Fig. 11). With the error set (a), centroid motion reaches $\sim 50 \mu \mathrm{m}$ and the resultant emittance growth reaches $\sim 300 \mathrm{pm}$. The corresponding values for the error set (b) are $\sim 25 \mu \mathrm{m}$ and $\sim 200 \mathrm{pm}$. If rf errors are maintained within $\pm 0.2^{\circ}$ phase errors and $\pm 0.1 \%$ voltage errors, better performance than the conventional scheme will be realized, because centroid oscillation due to injection system error in the conventional scheme is generally up to $50-100 \mu \mathrm{m}$. It should be also noted that the emittance blowup is temporary and damps down in 10000 turns (19.33 ms timescale), as shown in Fig. 11.

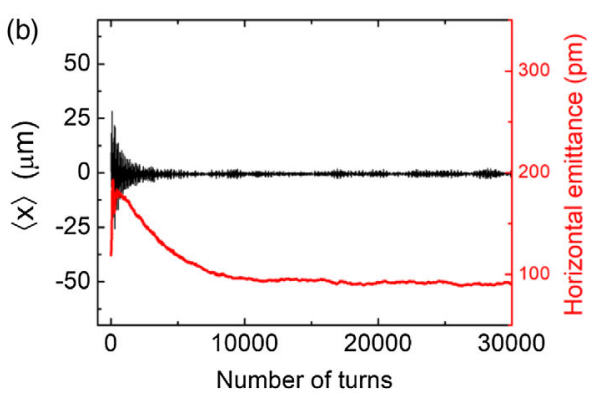

FIG. 11. Centroid oscillation (black line) and emittance growth (red line) along turn by turn for the cases of rf error with $0.2^{\circ}$ phase deviation and $0.1 \%$ voltage error (a) and rf error with $-0.2^{\circ}$ phase deviation and $-0.1 \%$ voltage error (b). The emittance blowup is temporary and damps down in 10000 turns (19.33 ms timescale). 


\section{CONCLUSION}

This paper presents and simulates a new on-axis top-up injection scheme for a fourth-generation storage ring. In the new injection scheme, longitudinal injection is implemented using two transverse deflecting cavities instead of using a short pulse kicker. A second transverse deflecting cavity kicks the injected beam toward on axis in transverse phase space. The first and second transverse deflecting cavities are operated to crab and uncrab the stored beam. In the numerical simulation and analysis, a transverse on-axis injection was realized without causing a remarkable disturbance in the stored beam along the storage ring. To get a better injection leakage impact (i.e., below 50-100 $\mu \mathrm{m}$ stored beam oscillation) by the conventional injection system, a phase deviation must be maintained within $\pm 0.2^{\circ}$ phase errors and $\pm 0.1 \%$ voltage error. A short $\mathrm{x}$-ray pulse can be generated from the bending magnet between two transverse deflecting cavities; this is an additional benefit of the new injection scheme.

\section{ACKNOWLEDGMENTS}

We thank M. Aiba (PSI) for providing helpful information and many useful discussions. This research was supported by POSTECH Basic Science Research Institute and by the Basic Science Research Program through the National Research Foundation of Korea (NRF-2015R1D1A1A01060049).

[1] MAX IV Detailed Design Report, https://www.maxiv.lu .se/accelerators-beamlines/accelerators/acceleratordocumentation/max-iv-ddr/.

[2] L. Liu, N. Milas, A. H. C. Mukai, X. R. Resende, A. R. D. Rodriguet, and F.H. Sa, in Proceedings of the 4th International Particle Accelerator Conference, IPAC2013, Shanghai, China, 2013 (JACoW, Shanghai, China, 2013), p. 1874.

[3] ESRF-EBS introduction, http://indico.psi.ch/conference Display.py? confId $=5589$.

[4] APS Upgrade introduction, https://www.aps.anl.gov/APSUpgrade.

[5] Spring-8-II conceptual design report, http://rsc.riken.jp/ eng/index.html.
[6] C. Steier et al., in Proceedings of the 7th International Particle Accelerator Conference, IPAC 2016, Busan, Korea, 2016 (JACoW, Geneva, 2016), p. 2956.

[7] M. Borland, A super-bright storage ring alternative to an energy recovery linac, Nucl. Instrum. Methods Phys. Res., Sect. A 557, 230 (2006).

[8] K. Tsumaki and N. Kumagai, Very low emittance light source storage ring, Nucl. Instrum. Methods Phys. Res., Sect. A 565, 394 (2006).

[9] A. Xiao, M. Borland, and C. Yao, in Proceedings of the 25th Particle Accelerator Conference, PAC-2013, Pasadena, CA, 2013 (IEEE, New York, 2013), p. 1076.

[10] A. Wolski, Low-emittance storage rings, CERN Accelerator School: Advanced Accelerator Physics Course (CERN, Geneva, 2014), pp. 245-294.

[11] M. Aiba, M. Böge, F. Marcellini, Á. Saá Hernández, and A. Streun, Longitudinal injection scheme using short pulse kicker for small aperture electron storage rings, Phys. Rev. ST Accel. Beams 18, 020701 (2015).

[12] A. Zholents, P. Heimann, M. Zolotorev, and J. Byrd, Generation of subpicosecond X-ray pulses using RF orbit deflection, Nucl. Instrum. Methods Phys. Res., Sect. A 425, 385 (1999).

[13] A. Zholents, A new possibility for production of subpicosecond x-ray pulses using a time dependent radio frequency orbit deflection, Nucl. Instrum. Methods Phys. Res., Sect. A 798, 111 (2015).

[14] Design study report: Generation of short pulses in SPEAR3 with two-frequency crab cavities, Report No. SLAC-R1089, 2016.

[15] M. Borland, Simulation and analysis of using deflecting cavities to produce short X-ray pulses with the Advanced Photon Source, Phys. Rev. ST Accel. Beams 8, 074001 (2005).

[16] RF Design of Normal Conducting Deflecting Cavity, https:/www-conf.slac.stanford.edu/icfa2010/proceedings/ FLS2010_normal_conducting_deflector_V_Dolgashev_ 4Mar2010.pdf.

[17] J. S. Jang (to be published).

[18] S. Shin et al., Commissioning of the PLS-II, J. Instrum., 8, P01019 (2013).

[19] M. Johansson, J. Langton, and J. Safranek, in Proceedings of the 9th International Particle Accelerator Conference, IPAC 2018, Vancouver, 2018 (JACoW, Geneva, 2018), p. 3831 .

[20] M. Borland, Elegant: A flexible SDDS-compliant code for accelerator simulation, Advanced Photon Source Report No. LS-287, 2000. 